University of Nebraska - Lincoln

DigitalCommons@University of Nebraska - Lincoln

\title{
Coxsackievirus B3 Infection Leads to the Generation of Cardiac Myosin Heavy Chain- $\alpha$-Reactive CD4 T Cells in A/J Mice
}

\author{
Arunakumar Gangaplara \\ University of Nebraska - Lincoln \\ Chandirasegaran Massilamany \\ University of Nebraska-Lincoln, cmassilamany@unl.edu \\ Deborah M. Brown \\ University of Nebraska - Lincoln, dbrown7@unl.edu \\ Gustavo A. Delhon \\ University of Nebraska - Lincoln, gdelhon3@Unl.edu \\ Asit K. Pattnaik \\ University of Nebraska - Lincoln, apattnaik2@unl.edu \\ See next page for additional authors
}

Follow this and additional works at: https://digitalcommons.unl.edu/vbsjayreddy

Part of the Cardiovascular Diseases Commons, Immunopathology Commons, Medical Microbiology Commons, Virology Commons, and the Virus Diseases Commons

Gangaplara, Arunakumar; Massilamany, Chandirasegaran; Brown, Deborah M.; Delhon, Gustavo A.; Pattnaik, Asit K.; Chapman, Nora; Rose, Noel; Steffen, David J.; and Reddy, Jay, "Coxsackievirus B3 Infection Leads to the Generation of Cardiac Myosin Heavy Chain- $\alpha$-Reactive CD4 T Cells in A/J Mice" (2012). Jay Reddy Publications. 1.

https://digitalcommons.unl.edu/vbsjayreddy/1

This Article is brought to you for free and open access by the Veterinary and Biomedical Sciences, Department of at DigitalCommons@University of Nebraska - Lincoln. It has been accepted for inclusion in Jay Reddy Publications by an authorized administrator of DigitalCommons@University of Nebraska - Lincoln. 


\section{Authors}

Arunakumar Gangaplara, Chandirasegaran Massilamany, Deborah M. Brown, Gustavo A. Delhon, Asit K. Pattnaik, Nora Chapman, Noel Rose, David J. Steffen, and Jay Reddy 
Published in Clinical Immunology (2012) 144: 237-249. DOI: 10.1016/j.clim.2012.07.003. Copyright 2012, Elsevier. Used by permission.

\title{
Coxsackievirus B3 Infection Leads to the Generation of Cardiac Myosin Heavy Chain-a-Reactive CD4 T Cells in A/J Mice Arunakumar Gangaplaraa, Chandirasegaran Massilamanya, Deborah M. Brown ${ }^{\mathrm{b}}$, Gustavo Del- hon ${ }^{\mathrm{a}}$, Asit K. Pattnaik ${ }^{\mathrm{a}}$, Nora Chapmanc, Noel Rose ${ }^{\mathrm{d}, \mathrm{e}, \mathrm{f}}$, David Steffena, Jay Reddy ${ }^{\mathrm{a},}$
}

\author{
aSchool of Veterinary Medicine and Biomedical Sciences, University of Nebraska-Lincoln, Lincoln, Nebraska, USA \\ bSchool of Biological Sciences, Nebraska Center for Virology, University of Nebraska-Lincoln, Lincoln, Nebraska, USA \\ 'Department of Pathology and Microbiology, University of Nebraska Medical Center, Omaha, Nebraska, USA \\ dDepartment of Pathology, Johns Hopkins Medical Institutions, Baltimore, Maryland, USA \\ eDepartment of Molecular Microbiology, Johns Hopkins Medical Institutions, Baltimore, Maryland, USA

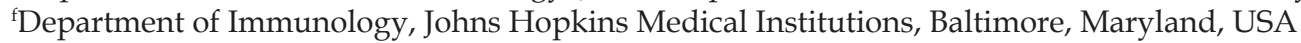 \\ *Corresponding author: jreddy2@unl.edu (J. Reddy).
}

Received March 27, 2012; accepted with revision July 3, 2012; available online July 14, 2012

Keywords: Autoimmunity; Autoreactive T cells; Cardiac myosin heavy chain-a; Coxsackievirus B3; Myocarditis; MHC class II dextramers

\begin{abstract}
Enteroviruses like coxsackievirus B3 (CVB3) are common suspects in myocarditis/dilated cardiomyopathy patients. Autoimmunity has been proposed as an underlying mechanism, but direct evidence of its role is lacking. To delineate autoimmune response in CVB3 myocarditis, we used IA ${ }^{k}$ dextramers for cardiac myosin heavy chain (Myhc)-a 334-352. We have demonstrated that myocarditis-susceptible A/J mice infected with CVB3 generate Myhc-a-reactive CD4 T cells and such a repertoire was absent in naïve mice as measured by proliferative response to Myhc-a 334-352 and IA ${ }^{\mathrm{k}}$ dextramer staining. We also detected Myhc-a 334-352 dextramer ${ }^{+}$cells in the hearts of CVB3-infected mice. The autoreactive T cell repertoire derived from infected mice contained a high frequency of interleukin-17-producing cells capable of inducing myocarditis in naïve recipients. The data suggest that CVB3, a bona fide pathogen of cardiovascular system that primarily infects the heart can lead to the secondary generation of autoreactive $\mathrm{T}$ cells and contribute to cardiac pathology.
\end{abstract}

Abbreviations: 7-AAD, 7-aminoactinomycin D; ANT, adenine nucleotide translocator; BCKD, branched chain a-ketoacid dehydrogenase; CPE, cytopathic effect; Con-A, concanavalin-A; cpm, counts per minute; CVB3, coxsackievirus B3; DCM, dilated cardiomyopathy; EAM, experimental autoimmune myocarditis; FC, flow cytometry; GM-CSF, granulocyte macrophage-colony stimulating factor; IFN, interferon; IL, interleukin; IRBP, interphotoreceptor retinoid-binding protein; LN, lymph nodes; MHC, major histocompatibility complex; MNC, mononuclear cells; Myhc-a, cardiac myosin heavy chain-a; NASE, neuraminidase; PI, postinfection; PMA, phorbol 12-myristate 13-acetate; RNase, ribonuclease; TCID50, 50\% tissue culture infective dose; Th, T helper; TNF, tumor necrosis factor; TNI, cardiac troponin I.

\section{Introduction}

Heart failure is the leading cause of death in the United States. It is estimated that $\sim 80$ million American adults have some form of cardiovascular disease, with a projected incidence of $40 \%$ by 2030 [1]. The potential causes of heart failure include pericardial, coronary artery, or heart valve diseases, idiopathic cardiomyopathy, chronic ischemia, and myocarditis.

Myocarditis is an inflammation of the myocardium, but only $\sim 10 \%$ of those affected show clinical manifestations of the disease [2]. Nonetheless, myocarditis is still regarded as an important cause of heart failure in children and adolescents, particularly athletes, indicating that a low degree of inflammation could be present in the hearts of apparently healthy individuals. This hypothesis was supported by a study of autopsies in more than 12,000 accidental deaths, which showed evidence of lymphocytic myocarditis in $1.06 \%$ of individuals [3], raising a question as to the trigger of inflammation in heart tissues. Furthermore, as a sequel to myocarditis, affected individuals can develop dilated cardiomyopathy (DCM) [4]. Approximately half of patients with DCM undergo heart transplantations due to the lack of effective chemotherapy. Enteroviruses are commonly suspected in DCM patients because the genomic material can be detected in up to $70 \%$ of patients, and serologically, virus-reactive neutralizing antibodies can be found in $\sim 50 \%$ of patients [2]. The question to be addressed is how virus infection can promote DCM. Autoimmunity is one possible mechanism, as evidenced in patients with myocarditis/DCM by the presence of autoantibodies to various cardiac antigens, such as cardiac myosin heavy chain (Myhc)- $\alpha$, adenine nucleotide translocator (ANT), $\beta$-adrenergic receptor-1, branched chain a-ketoacid dehydrogenase (BCKD), laminin, and muscarinic receptor; Myhc-a is a well-characterized antigen [5,6].

Based on cellular infiltrations, forms of myocarditis have been classified as lymphocytic, giant cell, and eosinophilic, and various infectious (viruses, bacteria, protozoa, helminthes) and noninfectious (drugs, metals, chemicals) agents 
have been implicated in the causation of myocarditis. Prominent among the infectious causes are viruses, importantly enteroviruses like coxsackievirus B3 (CVB3). To study the immune events of myocardial injuries in CVB3 infection, various rodent models have been developed. For example, the virus induces myocarditis in susceptible strains of $\mathrm{A} / \mathrm{J}(\mathrm{H}-$ $\left.2^{a}\right)$ and Balb/C $\left(\mathrm{H}-2^{\mathrm{d}}\right)$ mice, the histologic features of which resemble those in human disease [7].The disease course has two distinct stages that occur in continuum: the acute phase, in which infectious virus is present causing damage to cardiac myocytes; and the chronic phase, in which inflammation persists, although the extent of virus replication is much reduced due to selection of a defective virus $[8,9]$. This is consistent with the observation that infectious CVB3 cannot be isolated in cardiac tissues from patients with DCM $[2,10]$.

The long-standing question is whether this inflammatory process occurs due to an autoimmune response to cardiac antigens. Importantly, the same strains of mice that are susceptible to chronic myocarditis and DCM after viral infection also are susceptible to disease induction by immunization with Myhc-a. In contrast, the strains that develop only limited acute myocarditis are resistant to Myhc-a-induced disease [11]. In addition, susceptible mice made tolerant to Myhc-a fail to develop myocarditis after viral infection [12]. It was previously shown that lymphocytes isolated on day 7 postinfection (PI) from Balb/C mice infected with CVB3 were lytic to cardiomyocytes, but their putative target antigens were not characterized [13,14]. Furthermore, these cytotoxic lymphocytes induced the disease in naïve mice infected with or without CVB3 prior to the transfer [14,15]. While these observations point to a role for autoreactive $\mathrm{T}$ cells in the mediation of CVB3 myocarditis, it is critical to delineate both the mechanisms of their generation and their target antigens in cardiac tissues.

With the establishment of experimental autoimmune myocarditis (EAM) disease models induced with various cardiac antigens, it is now generally accepted that autoreactive CD4 $\mathrm{T}$ helper (Th) cells play a pivotal role in the mediation of autoimmune myocarditis [16]. The relevance of autoreactive CD4 T cells is further emphasized by the fact that histologic features of EAM closely mimic those of postinfectious myocarditis induced with CVB3 [6,8]. Therefore, to study the role of autoreactive CD4 T cells in the occurrence of CVB3 myocarditis, we recently created major histocompatibility complex (MHC) class II/IA ${ }^{\mathrm{k}}$ dextramers for Myhc-a 334352 , which permitted us to enumerate the frequencies of antigen-specific $\mathrm{T}$ cells in infected mice. Using these reagents, we demonstrated that $\mathrm{A} / \mathrm{J}$ mice infected with CVB3 develop myocarditis accompanied with the generation of Myhca-specific CD4 T cells and they infiltrate into the hearts of infected animals. We noted that the autoreactive $T$ cells obtained from CVB3-infected mice contain a significant proportion of interleukin (IL)-17-producing cells capable of inducing myocarditis in naïve mice.

\section{Materials and methods}

\subsection{Mice}

Six-to-eight-week-old A/J and B10.A mice were procured from the Jackson Laboratory (Bar Harbor, Maine). The mice were maintained according to the animal protocol guidelines of the University of Nebraska-Lincoln, Lincoln, Nebraska.
2.2. Virus propagation, titration, and disease-induction Vero cells (ATCC, Manassas, Virginia) were grown to 80 to $90 \%$ confluence in $75 \mathrm{~cm}^{2}$ flasks in EMEM supplemented with $10 \%$ fetal bovine serum (FBS). After removing the medium, the adherent monolayer of cells was infected with CVB3 (Nancy strain, ATCC) with multiplicity of infection 1 in EMEM containing no FBS [9]. The virus was allowed to adsorb to the cells by incubating the flasks at $37^{\circ} \mathrm{C}$ for $1 \mathrm{~h}$ with gentle intermittent rotations every 10 to 15 min. Maintenance medium containing $2 \%$ FBS was then added and incubation was continued. After confirming the cytopathic effect (CPE) of the virus, the infected culture flasks were subjected to three rounds of freezing and thawing, and the culture supernatants containing virus were harvested by centrifuging cell lysates at $6000 \mathrm{rpm}$ for $10 \mathrm{~min}$ at $4^{\circ} \mathrm{C}$, aliquoted, and stored at $-80^{\circ} \mathrm{C}$ until further use.

Fifty $\mu 1$ of Vero cells prepared to a density of $5 \times 10^{5}$ cells $/ \mathrm{ml}$ in EMEM/10\% FBS was added in eight replicates for each dilution of the virus, plus four replicates as controls, in 96-well plates, which was incubated at $37^{\circ} \mathrm{C}$ for $1 \mathrm{~h}$. Ten-fold serial dilutions of virus stock $\left(10^{-1}\right.$ to $\left.10^{-8}\right)$ were made in EMEM and $50 \mu \mathrm{l}$ of these suspensions were added into the corresponding replicates, whereas control wells received only EMEM. The plates were scored for CPE up to 7 days, and 50\% tissue culture infective dose (TCID50) values were determined according to the Reed-Muench method [17].

The virus stock with known titers was diluted in $1 \times$ phosphate-buffered saline (PBS) to contain 25 to $1 \times 10^{5}$ TCID50/100 $\mu \mathrm{l}$, and the virions were injected into A/J mice i.p., whereas control animals received $1 \times$ PBS only. The animals were monitored for clinical illness and/or mortalities, if any, and the surviving animals were sacrificed on day 21 postinoculation. Hearts and pancreases were harvested for histology, spleens and lymph nodes (LN) were collected for in vitro experimentation.

\subsection{Histopathology}

Hearts and pancreases were fixed by immersion in $10 \%$ phosphate buffered formalin, and three cross-sectional levels of each were sectioned in $5 \mu \mathrm{m}$ thickness and stained with hematoxylin and eosin ( $\mathrm{H}$ and $\mathrm{E})$ [18]. Hearts and pancreases were evaluated for inflammation and necrotic changes and scored for severity based on the numbers of inflammatory foci added across all sections. The degree of inflammation was scored as normal (0), mild multifocal (1-5 foci), moderate multifocal to coalescing (6-25 foci), and severe (26 or more foci) [19].

\subsection{Peptide synthesis}

Myhc-a-334-352 (DSAFDVLSFTAEEKAGVYK), cardiac troponin I (TNI157-171: QALLGTRAKESLDLR), interphotoreceptor retinoid-binding protein (IRBP 201-216: ADKDVVVLTSSRTGGV), and bovine ribonuclease (RNase) 43-56 (VNTFVHESLADVQA) were synthesized on 9-fluorenylmethyloxycarbonyl chemistry (Neopeptide, Cambridge, Massachusetts). All peptides were HPLC-purified (N 90\%), identity was confirmed by mass spectroscopy, and dissolved in 1 $\times$ PBS prior to use.

\subsection{T cell proliferation assay}

Single-cell suspensions were prepared from a pool of 
spleens and LN (hereafter termed lymphocytes) harvested from CVB3-infected mice on day 21 PI, and the cell pellets were resuspended in $1 \times$ ammonium chloride potassium buffer (Lonza, Walkersville, Maryland) to lyse erythrocytes as described [19]. Likewise, lymphocytes also were prepared from naïve mice. After washing, cell pellets were resuspended in RPMI medium supplemented with $10 \%$ FBS, $1 \mathrm{mM}$ sodium pyruvate, $4 \mathrm{mM}$ L-glutamine, $1 \times$ each of non-essential amino acids and vitamin mixture, and $100 \mathrm{U} / \mathrm{ml}$ penicillinstreptomycin (Lonza; hereafter called growth medium), and cell viability was verified by trypan blue staining. Cells werestimulated at a density of $5 \times 10^{6} \mathrm{cells} / \mathrm{ml}$ in triplicates with the indicated peptides $(0-100 \mu \mathrm{g} / \mathrm{ml})$ for 2 days in growth medium; the cells cultured with no peptides served as medium controls. Proliferative responses were measured as counts per minute $(\mathrm{cpm})$ after pulsing with tritiated ${ }^{3}[\mathrm{H}]$ thymidine $(1 \mu$ Ci per well; MP Biomedicals, Santa Ana, California) for $16 \mathrm{~h}$ using a Wallac liquid scintillation counter (Perkin Elmer, Waltham, Massachusetts) [20,21]. Stimulation indices were then derived by dividing the cpm values obtained in peptide-stimulated cultures by those of background values obtained in medium controls [19].

2.6. Determination of antigen specificity of Myhc-a-reactive T cells by IA ${ }^{\mathrm{k}} /$ dextramer staining

IA $^{k}$ dextramers for Myhc-a 334-352 and RNase 43-56 were derived as described previously [22]. Briefly, soluble $I^{k}{ }^{k}$ molecules containing the above peptide sequences were expressed in baculovirus and the proteins were biotinylated [23]. Multimerized dextramers were generated by mixing biotinylated $\mathrm{IA}^{\mathrm{k}}$ proteins with phycoerythrin-conjugated dextran molecules at a 1:20 molar ratio [22].To determine the frequency of antigen-specific cells, lymphocytes derived from CVB3-infected mice were stimulated with Myhc-a 334$352(100 \mu \mathrm{g} / \mathrm{ml})$ for 2 days and maintained in growth medium containing IL-2 (eBioscience, San Diego, California; Advanced Biotechnologies, Columbia, Maryland). Viable lymphoblasts were harvested on day 5 by Ficoll-Hypaque density gradient centrifugation, and the cells were maintained in IL-2 medium. The staining was performed on day 9 using IA $^{\mathrm{k}}$ /Myhc-a 334-352 or RNase 43-56 dextramers, anti-CD4 (eBioscience), and 7-aminoactinomycin D (7-AAD; Invitrogen, Carlsbad, California) as previously described [22,23]. After acquiring the cells by flow cytometry (FC; FACSCalibur, BD Biosciences, San Diego, California), percentages of dextramer-positive $\left(\right.$ dext $\left.^{+}\right)$cells were determined in the live (7-AAD-) $\mathrm{CD}^{+}$subset using Flow Jo software (Tree Star, Ashland, Oregon).

For enumeration of Myhc-a-specific cells ex vivo,CD4 T cells were enriched by magnetic separation (IMag, BD Biosciences, San Jose, California), and the cells were treated with neuraminidase (NASE; Type X from Clostridium perfringens, 0.7 units/ml, Sigma-Aldrich, St. Louis, Missouri) in serumfree $1 \times$ DMEM medium prior to dextramer staining, as we have described previously [21,23]. After washing once with $5 \mathrm{ml}$ of $1 \times$ PBS, cells were stained with $\mathrm{IA}^{\mathrm{k}}$ dextramers, anti-CD4, and 7-AAD, and dext ${ }^{+}$cells were analyzed in the live (7-AAD-) CD4 subset as above. To determine the frequencies of Myhc-a-reactive cells in the hearts, after the animals were euthanized, hearts were perfused with an injection of $10 \mathrm{ml}$ of $1 \times$ cold PBS into the left cardiac ventricles, then hearts were collected and minced. The tissue suspensions were incubated with a buffer containing $1 \times$ PBS, collagenase IV (Worthington, Lakewood, New Jersey), and 2\% FBS at $37^{\circ} \mathrm{C}$ for $15 \mathrm{~min}$ in a shaker with continuous agitation. The digested tissues were passed through an $18 \mathrm{G}$ needle and incubated for an additional $10 \mathrm{~min}$; cell pellets were obtained by centrifugation and resuspended in $40 \%$ buffered percoll. Cell suspensions were then overlaid with $75 \%$ percoll and centrifuged, and the interphase representing mononuclear cells (MNC) was collected and washed [24]. The identities of different subsets of MNC were verified by FC. Finally, MNC were stained with Myhc-a 334-352 or RNase 43-56 dextramers, anti-CD4, and 7-AAD, and frequencies of Myhca-specific cells were determined as above.

\subsection{Intracellular cytokine staining}

To enumerate the frequencies of cytokine-producing cells in naïve mice, lymphocytes were stimulated with phorbol 12-myristate-13 acetate (PMA: $20 \mathrm{ng} / \mathrm{ml}$ ) and ionomycin (300 $\mathrm{ng} / \mathrm{ml}$; Sigma-Aldrich) for $5 \mathrm{~h}$ in the presence of $2 \mathrm{mM}$ monensin (Golgi stop, BD Pharmingen, San Diego, California) $[20,25]$. After staining with anti-CD4 and 7-AAD, cells were fixed, permeabilized, and stained with anti-cytokine antibodies or isotype controls (eBioscience), and acquired by FC. Frequencies of cytokine-producing cells were then enumerated in the live (7-AAD-) $\mathrm{CD}^{+}$subset. Likewise, cytokineproducing cells from CVB3-infected mice were analyzed ex vivo as above. However, to determine the frequencies of cytokine-producing autoreactive T cells from CVB3-infected mice, lymphocytes were stimulated with Myhc-a 334-352 $(100 \mu \mathrm{g} / \mathrm{ml})$ for 2 days and the cultures were maintained in IL-2 medium. On day 5, cells were stimulated with PMA and ionomycin, and frequencies of cytokine ${ }^{+} \mathrm{CD} 4 \mathrm{~T}$ cells were determined as above. The clones of anti-cytokine antibodies used were: interferon (IFN)-ү (XMG1.2), IL-4 (11B11), IL-10 (JES5-16E3), IL-17A (eBio17B7), IL-17F (eBio18F10), granulocyte macrophage colony-stimulating factor (GM-CSF, MPI22E9), all from eBioscience; and IL-22 (140301; R\&D Systems, Minneapolis, Minnesota).

\subsection{Adoptive transfer myocarditis}

Groups of A/J mice were infected with CVB3 $\left(50 \mathrm{TCID}_{50}\right.$ / animal), and 21 days later, spleens, and LN were collected and single cell suspensions were prepared [26]. Cells were stimulated with Concanavalin-A (Con-A; $1 \mu \mathrm{g} / \mathrm{ml}$ ) for 2 days and viable lymphoblasts were harvested and transferred i.p., into whole body irradiated naïve A/J mice (400 rads/ animal; $12 \times 10^{7}$ cells/animal). Each mouse additionally received pertussis toxin (PT, 100 ng; List Biologicals, Campbell, California) on days 0 and 2 posttransfer as described previously $[19,27,28]$. The animals were euthanized on day 21 posttransfer, and hearts and pancreases were collected for histological evaluation of inflammatory changes. To address whether Myhc-a-reactive CD4 T cells generated in infected mice can transfer the disease, we first stimulated the lymphocytes obtained from CVB3-infected mice with Myhc-a 334-352 (100 $\mu \mathrm{g} / \mathrm{ml})$ for 2 days and the cultures were maintained in IL-2 medium. On day 5 poststimulation, CD4 T cells were isolated by magnetic separation, and after resting for 2 days in growth medium, cells were expanded with Con-A for $24 \mathrm{~h}$, and transferred into naïve mice $\left(0.18 \times 10^{7}\right.$ cells/animal $)$. The 
animals were euthanized on day 14 and the tissues were evaluated for histology. In addition, the animals that received saline and Con-A-stimulated lymphocytes obtained from naïve mice were used as controls $\left(0.5 \times 10^{7}\right.$ cells/animal; $\left.n=3\right)$. To provide specificity for CVB3-mediated myocarditis, we used influenza virus strain, A/Puerto Rico 8/34 (PR8) grown in the allantoic cavity of embryonated hen's eggs [29]. Groups of $\mathrm{A} / \mathrm{J}$ mice $(n=5)$ were infected intranasally with $500 \mathrm{egg}$ infectious units of virus in $30 \mu \mathrm{l}$ of $1 \times$ PBS under light isoflurane anesthesia and after 10 days, animals were euthanized to obtain lymphocytes from LN and spleens. Cells were stimulated with Con-A as above for 2 days and viable cells were transferred into naïve mice $\left(0.3 \times 10^{7}\right.$ cells/animal). Animals were euthanized on day 14 posttransfer and hearts and pancreases were harvested for histology. Likewise, to provide specificity for Myhc, adoptive transfer experiments were performed using lymphocytes sensitized with IRBP 201-216 as an irrelevant antigen. Briefly, A/J mice were immunized with IRBP 201-216, an IAk-binder [30] in complete Freund's adjuvant $(50 \mu \mathrm{g} /$ mouse: $n=2)$ and 10 days later, the animals were euthanized to harvest lymphocytes from LN and spleens. Cells were stimulated with Con-A for 2 days and viable cells were administered into naïve mice $\left(0.75 \times 10^{7}\right.$ cells/ animal), and on day 14, the animals were euthanized to collect tissues for histology.

\subsection{Detection of CVB3 RNA by RT-PCR}

Total RNA was isolated from heart and pancreas homogenates, Con-A-stimulated lymphoblasts used for adoptive transfer experiments, Vero cells cultured with the cell medium used for suspending the lymphoblasts or Vero cells infected with CVB3 using RLT buffer containing guanidium thiocyanate (RNeasy kit, Qiagen, Valencia, California), and the samples were treated with RNAse-free DNAse I according to the manufacturer's recommendations (Qiagen). To make certain that the RNA samples were free of residual DNA, a second round of DNAse digestion was performed using amplification grade DNAse I (Invitrogen), and cDNAs were synthesized utilizing Superscript III reverse transcriptase kit as recommended (Invitrogen). CVB3 viral protein (VP)1 fragment was then amplified by PCR using VP1-specific primers (forward, 5'TTGCATATGGCCCAGTGGAAG3'; reverse, 5'TGTGGATCCTTATTGCCTAGTAGTGGTAACTC3'). The PCR products were stained with ethidium bromide and resolved in $1 \%$ agarose gel electrophoresis.

\subsection{Statistics}

Differences in the $\mathrm{T}$ cell proliferative responses, frequencies of dext ${ }^{+}$cells and cytokine secretion between treatment groups were analyzed by Student's t. P $\leq 0.05$ values were considered significant.

\section{Results}

3.1. Establishment of CVB3-induced myocarditis in A/J mice We used myocarditis-susceptible $\mathrm{A} / \mathrm{J}$ mice to induce the disease with CVB3. Determination of an autoimmune response in this model required establishment of a disease course in which the virus does not induce significant mortalities. This was achieved using different amounts of virus inocula and assessing disease severity by evaluating hearts and pancreases for histological evidence of inflammatory changes. The dose of $50 \mathrm{TCID}_{50} /$ animal resulted in $20 \%$ mortality of the infected animals, whereas doses ranging from $1 \times 10^{3}$ to $1 \times 10^{5} \mathrm{TCID}_{50}$ led to high mortalities ( $89 \%$ to $100 \%$ ) as early as 5 to 14 days PI (Table 1 ). Regardless of mortalities, however, $\sim 50 \%$ of infected animals had myocardial necrosis, which occurred in association with pancreatitis, but the degree of inflammatory changes varied. For example, high viral doses $\left(1 \times 10^{3}\right.$ to $\left.1 \times 10^{5} \mathrm{TCID}_{50}\right)$ resulted in mild inflammatory heart lesions composed of fewer perivascular lymphocytes, but all animals had severe necrosis; on the other hand, at low doses the inflammatory foci were widespread $\left(50 \mathrm{TCID}_{50}\right.$ : $\left.11.90 \pm 4.50 ; 100 \mathrm{TCID}_{50}: 14.30 \pm 5.72\right)$, but necrosis was noted in only $34 \%$ of mice (Figure 1 and Table 1). Such an inverse relationship might be due to limited time available for immune events to set in as a result of early mortalities. However, necrosis and atrophic changes were evident in the pancreases of all infected animals (Figure 1). These features were lacking in tissues harvested from the control group (data not shown). Based on our data, we chose a dose range of 50 to $100 \mathrm{TCID}_{50} /$ animal for further experiments to investigate the induction of autoreactive $\mathrm{T}$ cells in infected mice.

3.2. A/J mice infected with CVB3 show generation of Myhca-reactive cells in their periphery

To address the hypothesis that CVB3 infection induces generation of autoreactive cells, we used Myhc- $\alpha$ as a putative target cardiac antigen [31]. Two approaches were adopted to evaluate the generation of Myhc-a-reactive CD4 T cells: 1 ) proliferative response to Myhc-a 334-352 based on ${ }^{3}[\mathrm{H}]$ thymidine incorporation assay, and 2) $\mathrm{IA}^{\mathrm{k}} /$ dextramer staining. Lymphocytes isolated from A/J mice infected with CVB3 on day 21 PI were tested for responses to Myhc-a 334-352 or RNase 43-56 (control). Figure 2A shows that the cells responded dose dependently to Myhc-a 334-352, and the response was specific since the cells did not respond to the control peptide. Stimulation indices obtained for each peptide revealed $\sim 2.5$-fold higher proliferative responses for Myhc-a 334-352 than for RNase 43-56. We next tested antigen specificity of these cells using $I \mathrm{~A}^{\mathrm{k}} /$ dextramers which permitted us to enumerate the frequencies of Myhc- $\alpha$-specific CD4 T cells by FC [22]. CD4 T cells were enriched from infected mice by magnetic separation and treated with NASE prior to dextramer staining [22]. NASE treatment is critical for ex vivo determination of antigen-specific cells since it facilitates efficient binding of class II tetramers or dextramers [23]. Under these conditions, we estimated the frequencies of Myhca-specific cells to be $0.1 \pm 0.003 \%$, whereas the staining intensity obtained with RNase $43-56$ dextramers was significantly low $(p=0.002$; Figure 2B). We verified these results by stimulating the lymphocytes harvested from CVB3-infected mice with Myhc-a 334-352, collecting lymphoblasts on day 9 poststimulation, and staining with dextramers. Myhc-a 334-352 dextramers detected $\sim 3$ times more $\mathrm{CD} 4$ cells than were detected with RNase $43-56$ dextramers $(0.46 \pm 0.08 \%$ vs. 0.14 $\pm 0.01 \% ; p=0.015$; Figure 2C). Expectedly, the number of Myhc-a-reactive cells detected in Myhc-a 334-352-stimulated cultures increased by $\sim 5$-fold over ex vivo analysis. The specificity of $\mathrm{T}$ cell responses to Myhc-a 334-352 was further verified by stimulating the lymphocytes obtained from CVB3-infected mice with a non-myocarditogenic heart anti- 
gen, TNI 157-171 [32]. Figure 2D shows that the lymphocytes obtained from CVB3-infected mice did not respond to TNI 157-171 and the response was similar to control, RNase 4356. Finally, we asked whether Myhc-a-reactive $\mathrm{T}$ cells can be generated in myocarditis-resistant mice. To address this question, we used B10.A mice bearing $\mathrm{H}-2^{\text {a }}$ haplotype similar to A/J mice which are resistant to the development of myocarditis induced with CVB3 $[33,34]$. In a T cell proliferation assay, we noted that lymphocytes obtained from infected B10.A mice did not respond to Myhc-a 334-352 (Figure 2E). Overall, the data obtained with dextramer stainings and proliferative responses corroborate each other and provide evidence for the generation of Myhc-a-reactive cells in mice susceptible to CVB3 infection.

3.3. Myhc-a-specific cells infiltrate into the hearts of CVB3infected mice

We sought to verify whether Myhc-a-specific cells infiltrate the hearts. MNC were harvested from hearts of CVB3-infected mice and stained with Myhc-a 334-352 or RNase 43-56 dextramers, and frequencies of Myhc-a-dext ${ }^{+}$cells were determined by FC. We prepared pools of MNC from at least seven hearts for each experiment because the yield of cells per heart was low $\left(6 \times 10^{4} /\right.$ heart $)$. The majority of infiltrates were non- $\mathrm{T}$ cells, and staining for different immune cell markers revealed the presence of $\mathrm{B}^{2} 20^{+}(8.5 \%), \mathrm{GR} 1^{+}(6.5 \%)$, $\mathrm{CD}_{11 b^{+}}(2.8 \%)$, and CD11c cells $(1.4 \%)$. In contrast, $\mathrm{CD}^{+} \mathrm{T}$ cells formed a minor component consisting of $\mathrm{CD}^{+}(8 \%)$ and $\mathrm{CD}^{+}(4 \%) \mathrm{T}$ cells (Figure 3A). Dextramer analysis of CD4 T cells revealed autoreactive cells as evidenced by the presence of an estimated 2.2 $\pm 0.16 \%$ of Myhc-a 334-352 dext ${ }^{+}$cells, as opposed to $0.6 \pm 0.07 \%$ of RNase $43-56$ cells, an $\sim_{\text {four-fold }}$ difference $(p=0.011$, Figure 3B). The data provide evidence that Myhc-a 334-352-reactive CD4 T cells infiltrate the hearts during the course of CVB3 infection in A/J mice.

3.4. Naïve A/J mice do not carry Myhc-a-reactive CD4 T cells in their peripheral repertoires

One critical question we needed to address was whether Myhc-a-reactive cells generated in CVB3-infected mice arise from expansion of a preexisting repertoire of autoreactive cells or appear as a new population of cells after viral infection. We obtained lymphocytes from naïve (uninfected) A/J mice and evaluated their reactivity to Myhc-a 334-352 by $\mathrm{T}$ cell proliferation assay and dextramer staining. Using RNase 43-56 as control, we noted that $\mathrm{T}$ cells did not respond to Myhc-a 334-352 (Figure 4A) nor was there any staining with Myhc-a dextramers (Figure 4B). Absence of Myhc-a-reactive cells in the naïve repertoires but their presence in CVB3-in- fected animals implies that autoreactive T cells appear secondary to virus infection, likely as the result of virus-induced tissue damage.

3.5. Myhc-a-reactive CD4 Tcells generated in response to CVB3 infection contain a high frequency of IL-17-producing cells

To determine the pathogenic potential of Myhc-a-reactive cells generated in mice infected with CVB3, we determined their cytokine-producing abilities by intracellular staining. Frequencies of cytokine-producing cells were enumerated in naïve mice to determine background response of cytokine production prior to exposure to the virus. The naïve $\mathrm{T}$ cell repertoires contained predominantly IL-2-producing cells (data not shown), whereas cells capable of producing other cytokines except IFN-ץ were lacking (1.25 $\pm 0.03 \%$; Figure 5). Examination of cytokine-producing abilities of lymphocytes harvested from CVB3-infected mice ex vivo revealed elevated levels of all cytokines tested - Th1, Th2, and Th17 - but among these, frequencies of cells secreting IL-22 (2.75 \pm $0.34 \% ; p=0.002)$ and GM-CSF $(0.36 \pm 0.03 \% ; p=0.006)$ were significantly increased, followed by cells producing IFN- $\gamma$


$(0.24 \pm 0.07 \%)$, and IL-4 (0.19 $\pm 0.08 \%$; Figure 5$)$. Next, we evaluated the frequencies of cytokine-secreting autoreactive $\mathrm{T}$ cells by stimulating lymphocytes harvested from CVB3-infected mice with Myhc-a 334-352, resulting in the expansion of IFN- $\gamma^{-}$, IL-4-, IL-17A-, IL-17F-, and GM-CSF-producing cells, but the prominent cytokine was IL-17A $(0.51 \pm 0.04 \%$, $\mathrm{p}=0.033$; Figure 5). The data suggest that Myhc-a-reactive cells generated in CVB3-infected mice possess the pathogenic characteristics of autoreactive $\mathrm{T}$ cells as indicated by their preferential ability to produce IL-17.

3.6. T lymphocytes generated from CVB3-infected mice can induce myocarditis in naïve mice

To determine if the autoreactive cells generated in mice infected with CVB3 have an ability to induce myocarditis, we derived lymphoblasts from infected mice by stimulating lymphocytes with Con-A and injecting them into naïve irradiated recipients as previously described [35,36]. One of the three recipients of cells derived from CVB3-infected mice showed epicarditis (Figure 6A: i), myocarditis (Figure 6A: ii) and myocardial fibrosis (Figure 6A: iii) and the pancreas was not affected and the infiltrates were predominantly composed of lymphocytes similar to EAM [19]. Likewise, the animals (2/2) that received Myhc-a 334-352-sensitized CD4 $T$ cells also showed cardiac damage as indicated by hemorrhagic necrosis (Figure 6B: i) and mineralization (Figure 6B: ii). On the contrary, none of the saline-receiving

Table 1 CVB3-induced myocarditis in A/J mice.

$\begin{array}{lcc}\text { Does }\left(\mathrm{TCID}_{50} / \text { animal }\right. & \text { Incidence n }(\%) & \text { Mortality n }(\%) \\ 2.5 \times 10^{1} & & \\ 5.0 \times 10^{1} & 1 / 3(33.33) & 1 / 3(33.33) \\ 1.0 \times 10^{2} & 12 / 55(21.81) & 11 / 55(20) \\ 1.0 \times 10^{3} & 13 / 33(39.39) & 22 / 35(62.85) \\ 1.0 \times 10^{4} & 5 / 9(55.55) & 9 / 9(100) \\ 1.0 \times 10^{5} & 4 / 9(44.44) & 8 / 9(88.88) \\ & 5 / 8(62.5) & 8 / 8(100)\end{array}$

\begin{tabular}{lll}
\multicolumn{2}{l}{ Severity of lesions } \\
\hline Mild & Moderate & Severe \\
0 & 1 & 0 \\
3 & 3 & 6 \\
6 & 2 & 5 \\
3 & 2 & 0 \\
4 & 0 & 0 \\
4 & 1 & 0
\end{tabular}

Inflammatory foci (mean \pm SEM)

$66.66 \pm 6.66$

$11.90 \pm 4.49$

$14.30 \pm 5.72$

$2.55 \pm 1.36$

$1.13 \pm 0.52$

$2.00 \pm 0.93$

Inflammation scoring scale: mild multifocal: 1-5 foci; moderate multifocal to coalescing: 6-25 foci; and severe: 26 foci and above. 


\section{Heart}
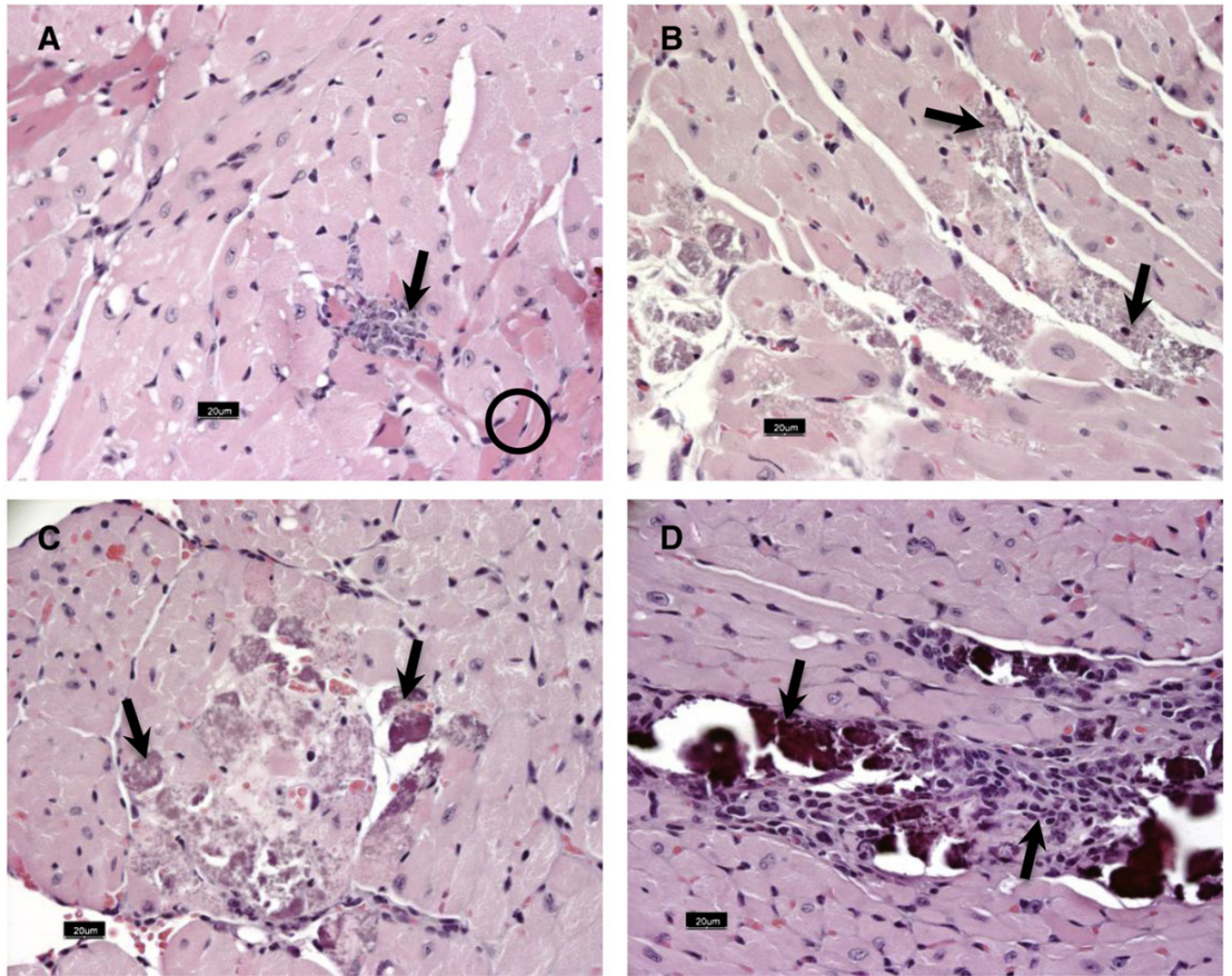

\section{Pancreas}
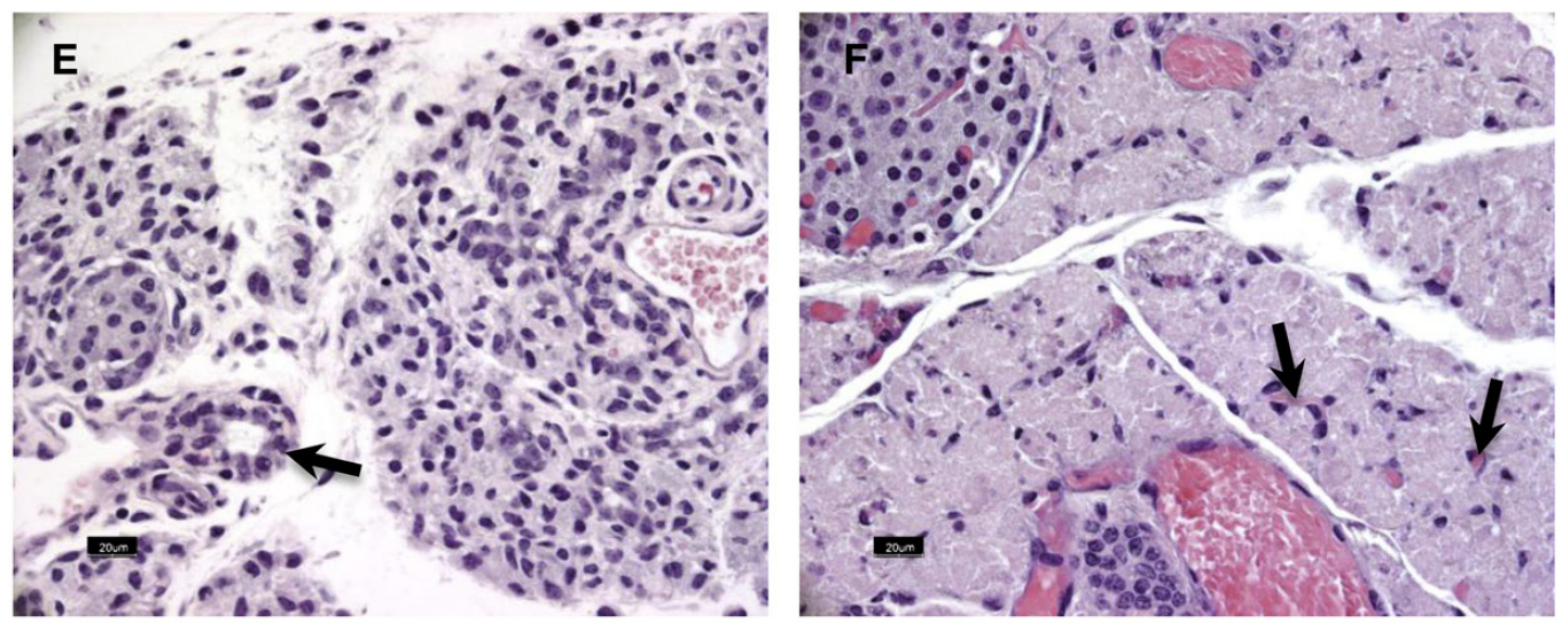

Figure 1 Histological evaluation of hearts and pancreases from mice infected with CVB3. Groups of 6- to 8-week old A/J mice were infected with CVB3 $\left(1 \times 10^{2} \mathrm{TCID}_{50} /\right.$ animal) i.p., and animals were killed on day 21 PI. Hearts (A to D) and pancreases (E and F) were collected for histological evaluation of inflammatory changes by $\mathrm{H}$ and E staining. (A) Mild lesion with a few perivascular lymphocytes (arrow) and dark pink fibers indicative of necrosis (circle). (B) Moderate lesions with more foci and necrosis (arrows). (C) Severe inflammatory lesion with widespread areas of mineralization (arrows). (D) Necrosis, mineralization, or lymphocytic and histiocytic infiltrates (arrows). (E) Pancreatic ducts (arrow) and small acinii composed of atrophic acinar cells and (F) widespread acinar cell necrosis with nuclear pyknosis (arrows) and loss of cytoplasmic details. 


\section{A) Proliferative response}
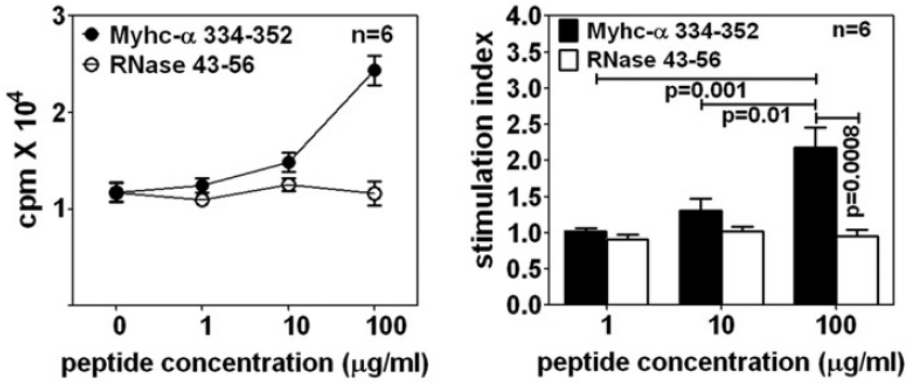

B) Dextramer staining ex vivo
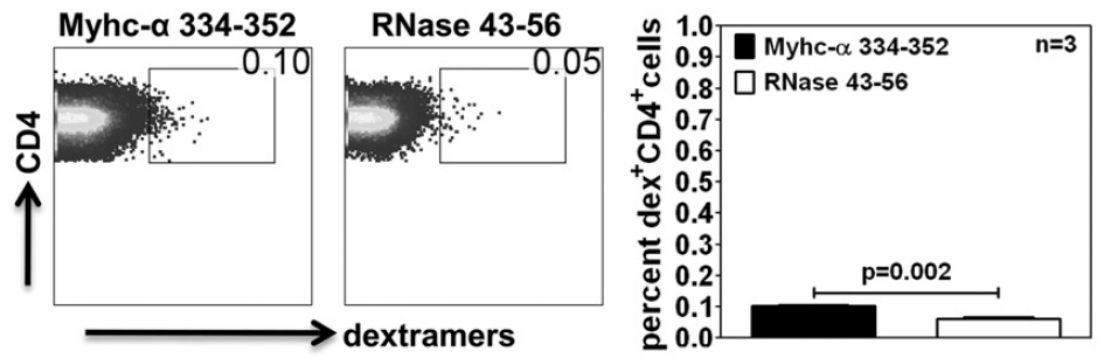

C) Dextramer staining of Myhc- $\alpha$ 334-352-stimulated cells
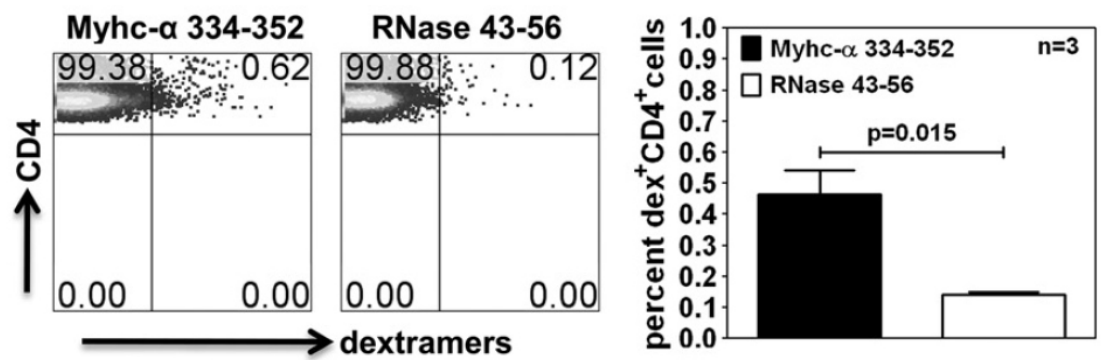

D) Proliferative response to TNI 157-171

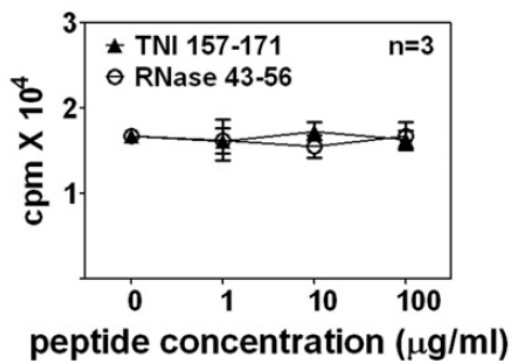

E) Proliferative response to Myhc- $\alpha$ 334-352 in B10.A mice

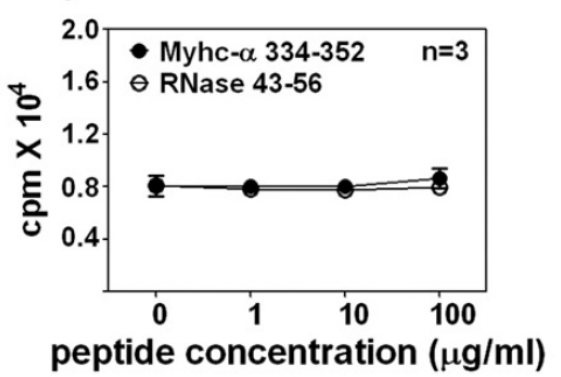

Figure 2 A/J mice infected with CVB3 show the generation of Myhc-a 334-352 reactive cells. (A) Proliferative response. A/J mice were infected with CVB3 $\left(5 \times 10^{1}\right.$ to $1 \times 10^{2} \mathrm{TCID}_{50} /$ animal); after 21 days, animals were sacrificed and spleens and LN were harvested to prepare lymphocytes. Cells were stimulated with Myhc-a 334-352 for 2 days, then pulsed with ${ }^{3}[\mathrm{H}]$ thymidine for $16 \mathrm{~h}$, and proliferative responses were measured as cpm. (B) Dextramer staining ex vivo. CD4 T cells isolated from CVB3-infected mice were treated with NASE prior to staining with IA ${ }^{\mathrm{k}} /$ dextramers, anti-CD4, and 7-AAD. After acquiring the cells by FC, dext ${ }^{+}$cells were analyzed in the live (7-AAD-), CD4 subset. Control, RNase 4356. (C) Dextramer staining of Myhc-a 334-352-stimulated cells. Lymphocytes were obtained from CVB3-infected mice as above; cells were stimulated with Myhc-a 334-352 for 2 days and cultures were maintained in IL-2 medium. Viable lymphoblasts were harvested on day 9 poststimulation and stained with $\mathrm{IA}^{\mathrm{k}}$ / dextramers, anti-CD4 and 7-AAD. After acquiring the cells by FC, dext ${ }^{+}$cells were analyzed in the live (7-AAD-), CD4 subset. Control, RNase 43-56. (D) Proliferative response to TNI 157-171. Lymphocytes harvested from CVB3-infected A/J mice were stimulated with TNI 157-171 and RNase 43-56 for 2 days and the proliferative responses were measured as cpm after pulsing with ${ }^{3}[\mathrm{H}]$ thymidine for 16 h. (E) Proliferative response to Myhc-a 334-352 in B10.A mice. B10.A mice were infected with CVB3 $\left(1 \times 10^{2} \mathrm{TCID}_{50} / \mathrm{mouse}\right)$ and after 21 days, the animals were euthanized to prepare single cell suspensions from LN and spleens. After stimulating the cells with Myhc-a 334-352 and RNase 43-56 for 2 days, and pulsing with ${ }^{3}[\mathrm{H}]$ thymidine, proliferative responses were measured as above. 
animals (Figure 6C), or those that received Con-A-stimulated naïve lymphocytes (Figure 6D), influenza virus-sensitized lymphocytes (Figure 6E) and IRBP 201-216-reactive lymphocytes (Figure 6F) showed any evidence of inflammation in the hearts or in the pancreases. To verify whether myocarditis occurring in the recipient mice was caused by residual virus present in the infused cells, we tested the lymphocyte cultures used for infusions for the presence of virus by CPE, and for the presence of viral nucleic acids by RT-PCR. Vero cells infected with CVB3 as a positive control showed cytolysis, but such a phenomenon was absent when the culture medium was passed thrice blindly, including the viral nucleic acids as verified for CVB3 VP1 (819 bp) by RT-PCR (lane, 14; Figure 7). Likewise, the presence of CVB3 RNA was not revealed in the evaluation of hearts and pancreases of mice



B) $I A^{k} /$ dextramer staining
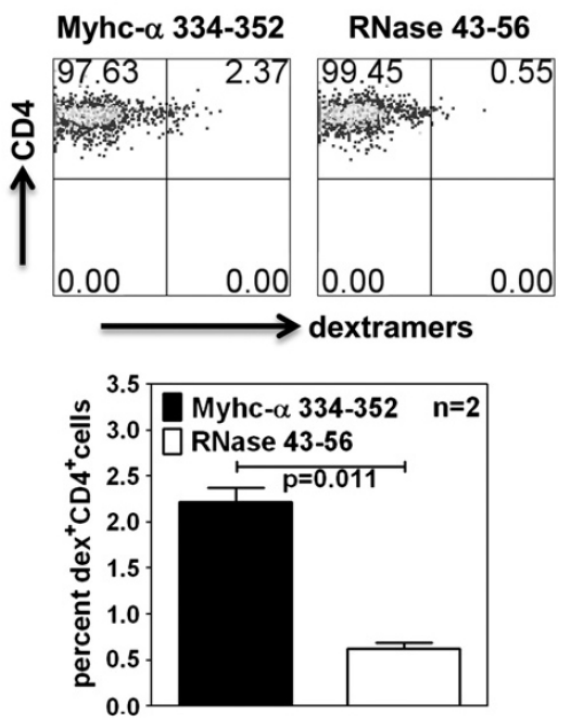

Figure 3 Myhc- $\alpha$-specific CD4 T cells infiltrate into the hearts of CVB3-infected mice. (A) Subsets of heart infiltrates. Groups of mice infected with CVB3 $\left(5 \times 10^{1} \mathrm{TCID}_{50} /\right.$ animal) were sacrificed on day 21 $\mathrm{PI}$, and hearts were collected to isolate MNC. Cells were stained with the indicated markers and percentages of each were determined by FC. The data represent an experiment involving 13 mice. (B) IA ${ }^{\mathrm{k}} /$ dextramer staining. Heart MNC isolated from CVB3-infected mice were stained with Myhc-a 334-352 or RNase 43-56 (control) dextramers and anti-CD4 and 7-AAD. Cells were acquired by FC, and percentages of dext ${ }^{+}$cells were enumerated in the live CD4 subset. Representative data from two individual experiments, each involving 7 to 8 mice, are shown. that received saline (lanes, 1-6) or cells (lanes, 7-12; Figure $7)$. The data suggest that CVB3 infection can lead to the generation of Myhc-a-reactive cells and they can contribute to the development of myocarditis in infected animals.

\section{Discussion}

In this report, we show that the $\mathrm{T}$ cell repertoire generated in response to CVB3 infection contains a population of Myhca-reactive cells. We provide four lines of evidence for a proposal that the autoreactive cells are generated secondary to CVB3 infection and play a role in the mediation of viral myocarditis: a) the peripheral repertoire of infected but not naïve mice contains a population of Myhc-a 334-352 dext ${ }^{+}$cells; b) the inflammatory infiltrates of hearts from CVB3-infected mice reveal the presence of Myhc-a-reactive cells; c) lymphocytes stimulated with Myhc-a 334-352 contain high frequencies of IL-17-producing cells, a cytokine generally implicated in various organ-specific diseases including autoimmune and CVB3 myocarditis or DCM [37-39]; and d) T cells generated from CVB3-infected mice can induce disease in naïve mice reminiscent of autoimmune myocarditis induced with Myhc-a 334-352 [16,19]. All these studies employed A/J mice, which are highly susceptible to both human-isolate CVB3 (Nancy strain) infection, and Myhc-a 334-352-induced EAM, whose disease pathologies resemble each oth-

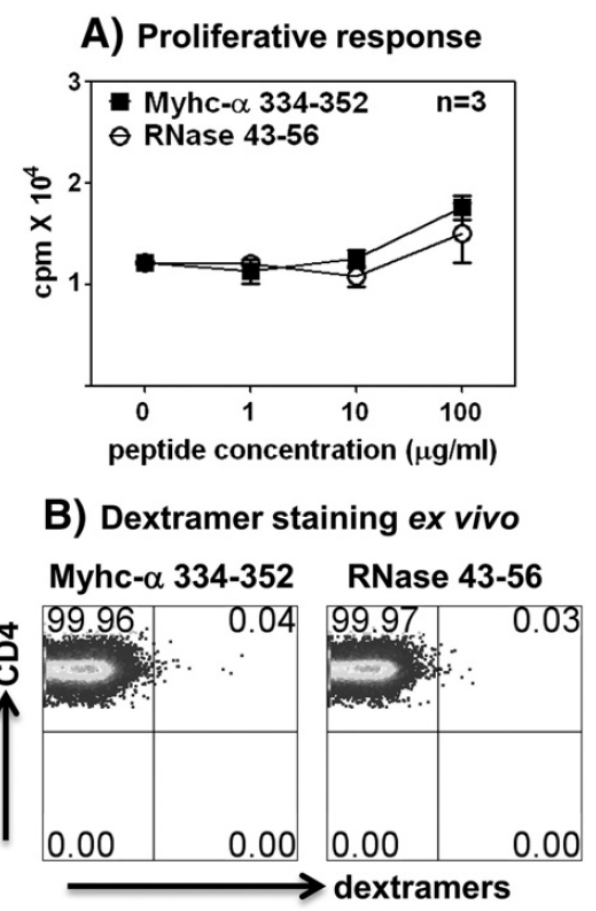

Figure 4 Naïve mice do not carry Myhc-a 334-352-reactive T cells in their periphery. (A) Proliferative response. Lymphocytes were isolated from spleens and LN harvested from naïve mice, and cells were stimulated with Myhc-a 334-352 or RNase 43-56 for 2 days; after pulsing with ${ }^{3}[\mathrm{H}]$ thymidine for $16 \mathrm{~h}$, proliferative responses were measured as cpm. (B) Dextramer staining ex vivo. CD4 T cells isolated from naïve mice were treated with NASE prior to staining with $\mathrm{IA}^{\mathrm{k}} /$ dextramers followed by anti-CD4 and 7-AAD. After acquiring the cells by $\mathrm{FC}$, dext ${ }^{+}$cells were analyzed in the live (7-AAD), CD4 subset. Control, RNase 43-56. FC plots representing three individual experiments each involving three mice are shown. 
er [11]. Therefore, the CVB3-induced myocarditis model provides a useful framework to extrapolate the observations made in mice to humans.

Autoimmune theory has been proposed in the pathogenesis of CVB3 infection, particularly in the heart, because the disease assumes a biphasic pattern: acute or viremic and chronic or non-viremic phases, although in the latter phase, the virus can persist but in a defective form [8,9]. Generally, autoimmunity becomes a prime suspect in chronic diseases when inflammation persists in the absence of detectable infectious particles. The challenge, then, is to provide support for the concept that autoimmunity is a component of the disease process to establish a cause-and-effect relationship.

Extensive investigation of the role of cell-mediated immune response in CVB3 infection has shown that it requires participation of both CD4 and CD8 T cells. Mice deficient in CD4 become resistant to CVB3-induced myocarditis, whereas CD8-deficient mice develop more or less severe myocarditis $[40,41]$. Conversely, mice deficient in both CD4 and CD8 are better protected from the disease [40]. In addition, $\gamma \delta \mathrm{T}$ cells promote susceptibility to CVB3-induced myocarditis by potentiating the expansion of Th1 cells [15]. These observations indicate that multiple immune cells are involved in the mediation of viral myocarditis. Generally, T cell-mediated organ-specific autoimmune diseases such as experimental autoimmune encephalomyelitis/multiple sclerosis are primarily driven by Th1 and Th17 cells [42]. An exception to this paradigm was earlier proposed to be myocarditis, in that Th1 cells offer disease protection. For example, IFN- $\gamma$ knockout mice become susceptible to CVB3-myocarditis, and treatment with IFN- $\gamma$ ameliorates the disease [43]. Likewise, tissue-specific ectopic expression of IFN-. within the pancreatic beta cells prevents the disease [44]. In contrast to Th1

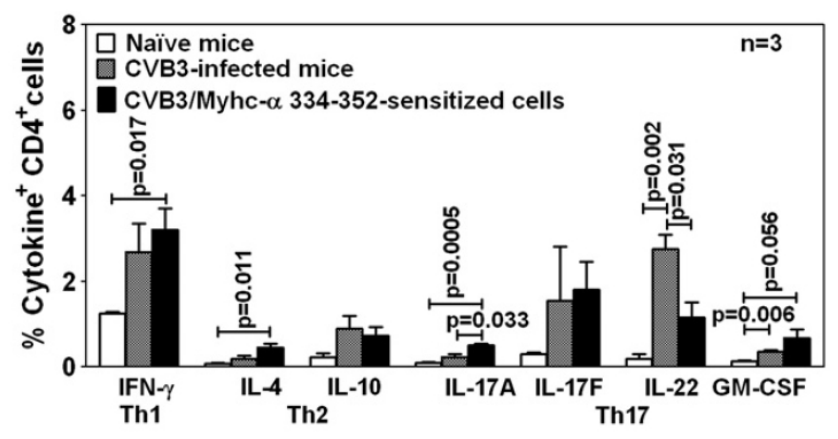

Figure 5 Myhc-a 334-352-sensitized CD4 T cells from CVB3- infected mice contain a high proportion of IL-17-producing cells. Lymphocytes harvested from naïve mice or from CVB3-infected mice on day 21 PI were stimulated with PMA and ionomycin in the presence of monensin for $5 \mathrm{~h}$. After staining with anti-CD4 and 7-AAD, cells were fixed and permeabilized followed by incubation with a panel of Th1, Th2 and Th17 cytokine antibodies. Cells were acquired by FC and frequencies of cytokine-producing cells were determined in the live (7-AAD-), CD4 subset. To determine the frequencies of cytokineproducing autoreactive $\mathrm{T}$ cells, lymphocytes obtained from CVB3infected mice were stimulated with Myhc-a 334-352 for 2 days; cultures were maintained in IL-2 medium. Viable lymphoblasts harvested on day 5 were stimulated with PMA/ionomycin and percentages of cytokine-producing cells were determined as above. cells, the role of IL-4- and IL-10-producing Th2 cells is more complex. Mice infected with CVB3 produce IL-4, which can dampen the disease-protective Th1 response. IL-4 also can suppress the production of innate inflammatory mediators, such as metalloproteinases, and favors recovery from viral myocarditis [45]. On the contrary, macrophages or human fibroblasts exposed to CVB3 produce both anti-inflammatory (IL-10) and inflammatory [IL- ${ }_{\beta}$, IL-6 and tumor necrosis factor (TNF)-a] cytokines, and induction of IL-10 is thought to favor development of the chronic disease course by inhibiting the disease-protective IFN-ץ secretion [46,47]. Recently, it was shown that IL-17 promotes viral myocarditis by enhancing viral replication and that neutralization of IL-17 using cytokine-specific antibody treatment suppresses the severity of disease induced with CVB3 [39]. We observed that lymphocytes derived from CVB3-infected mice contain a significant proportion of IL-22 $(2.75 \pm 0.34 \%)$ and GM-CSF $(0.36 \pm 0.03 \%)$. Similar profiles have been earlier reported [48]. However, after stimulation with Myhc-a 334-352, frequencies of IL-17-producing cells were increased suggesting a selective expansion of Th17 cytokine-secreting cells by an autoantigen. We and others have previously demonstrated that the lymphocytes generated from Myhc-a 334-352-immunized mice contain predominantly IL-17-producing cells $[4,19,37]$. Thus our data support the contention that IL-17 is a myocarditogenic cytokine [49], and IL-17-producing autoreactive $\mathrm{T}$ cells could be generated as a secondary event in infectious myocarditis and contribute to cardiac damage.

CVB3 is a cytolytic cardiotropic virus that causes extensive damage in the target organ, the heart. The dosages used in this study were low compared to other models of CVB3-induced myocarditis due to the high rate of mortality in the $\mathrm{A} / \mathrm{J}$ mice with this viral strain $[14,50]$. At dosages of $10^{3}$ to $10^{5}$ infectious units, mortality was greater than $80 \%$, whereas low doses (up to $1 \times 10^{2}$ ) induced myocarditis with low mortalities. Cardiovirulent CVB3 causes extensive pancreatic necrosis, which may account for the early mortalities in these mice [51]. We attempted to determine whether myocarditis that occurred in CVB3-infected mice might be due to the generation of autoreactive CD4 cells, and our data support this possibility. We noted that Myhc-a-reactive cells are generated in CVB3-infected but not naïve A/J mice $(0.1 \%$, Figure $2 \mathrm{~B}$ vs $0.04 \%$, Figure $4 \mathrm{~B}$ ) and antigen-specific T cells infiltrate into the hearts of infected animals. The absence of such a repertoire in naïve mice suggests their appearance secondary to virus infection. Whether naïve $\mathrm{A} / \mathrm{J}$ mice carry $\mathrm{T}$ cell repertoires for other epitopes of Myhc-a remains to be tested. This possibility seems less likely because Myhc-a 334352 was identified as the only immunodominant and pathogenic epitope in this mouse strain [16]. These observations, however, do not rule out the possibility that virus-specific cells are not involved. The question is: how do they mediate cardiac damage in the absence of replicating viral particles? This can be explained by two scenarios: 1) Virus-specific cytotoxic lymphocytes may expand from a pool of memory $\mathrm{T}$ cell population during subsequent exposures to CVB3, and the effector cells thus derived can kill virus-infected cardiomyocytes through MHC class I-dependent pathways. Likewise, Th cells capable of secreting inflammatory cytokines also can potentially expand and contribute to tissue injury or can do so through the production of autoantibodies. In sup- 
A) Con-A-stimulated lymphocytes from CVB3-infected mice
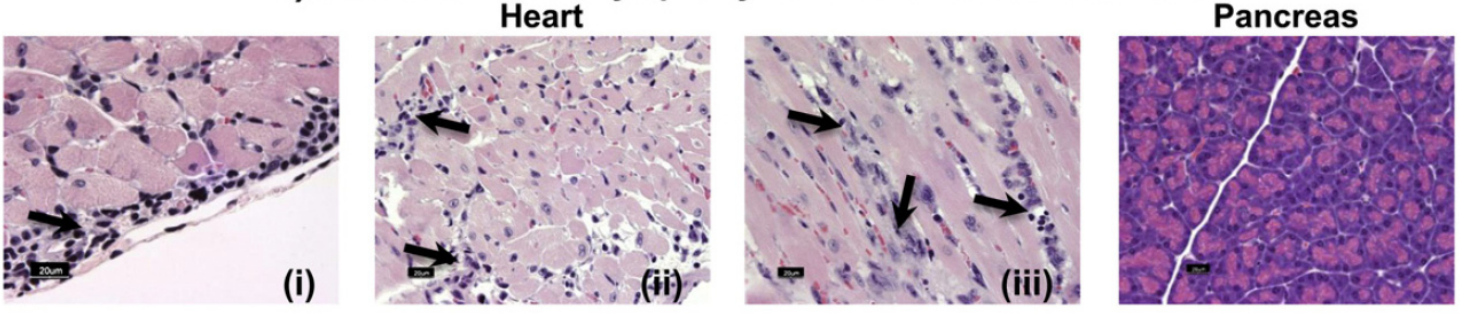

\section{B) Myhc- $\alpha$ 334-352-stimulated CD4 T cells from CVB3-infected mice \\ Pancreas}

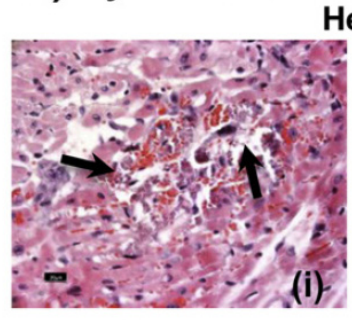

C) Saline
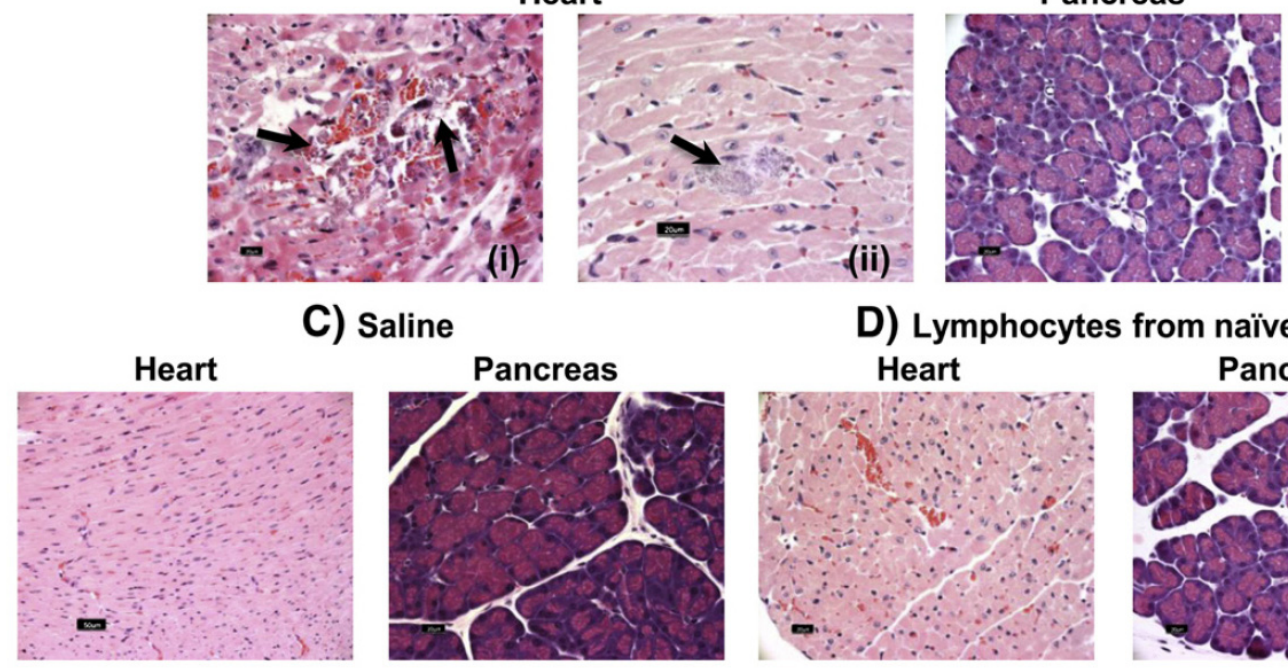

D) Lymphocytes from naïve mice

E) Lymphocytes from influenza virus-infected mice
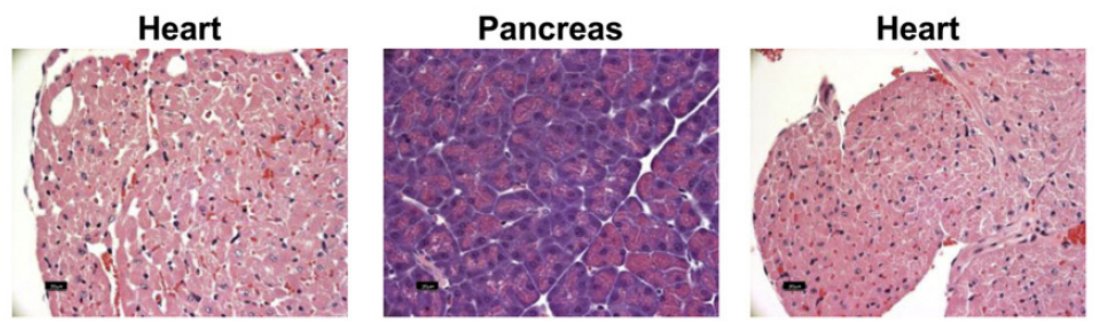

F) IRBP 201-216-reactive cells

Figure 6 T cells generated from CVB3-infected mice can induce myocarditis in naïve mice. Groups of A/J mice $(n=20)$ were infected with CVB3 and after 21 days, lymphocytes were stimulated with Con-A for 2 days, viable lymphoblasts were administered i.p., into the naïve irradiated mice $(n=3)$, and each mouse also received PT i.p. The animals were euthanized on day 21 posttransfer, and hearts and pancreases were collected for histological evaluation of inflammatory changes by $\mathrm{H}$ and $\mathrm{E}$ staining. Panels (A: i), (A: ii), and (A: iii) represent heart sections from mice that received the cells, respectively denoting epicarditis, myocarditis, myocardial fibrosis accompanied with inflammatory infiltrates (arrows), and normal pancreas. Likewise, Myhc-a 334-352-sensitized CD4 T cells were also generated from CVB3-infected mice as described in the Materials and methods and transferred into naïve mice. Panels (B: i) and (B: ii) respectively represent hemorrhagic necrosis and mineralization in the hearts (arrows) but with normal pancreas. Panels (C), (D), (E) and (F) represent histological evaluation of hearts and pancreases obtained from animals that received saline, Con-A-stimulated naïve lymphocytes, influenza virus-sensitized lymphocytes and IRBP 201-216-reactive cells generated from A/J mice. Bar=20 $\mu \mathrm{m}$ for all except for heart in saline group (C): $50 \mu \mathrm{m}$.

port of this theory, mice infected with repeated bouts of virus developed dilated hearts in association with increased TNF-a secretion and CVB3-reactive antibodies, but with minimal inflammatory cell infiltrations [52]. 2) Virus-specific cells may contain repertoires of cells specific to cardiac proteins other than Myhc, and existence of such cross-reactive T cell repertoires, if any, can induce cardiac injury through antigenic mimicry [53].

In summary, we provide evidence that CVB3-infected mice show the generation of Myhc-a-reactive cells and they infil- trate into the hearts of infected animals. Because the naïve mice lack Myhc-a-reactive cells in their periphery, we propose that generation of autoreactive $T$ cells occurs as a secondary event to cardiac damage. This means previously invisible self-proteins, as in the case of Myhc-a, become targets for immune attack after viral damage. While our data provide a proof-of-concept for this theory, we cannot rule out the contribution of autoreactive $T$ cells for other self-antigens that might be released during the course of CVB3 infection as a result of epitope spreading [54]. To investigate this pos- 


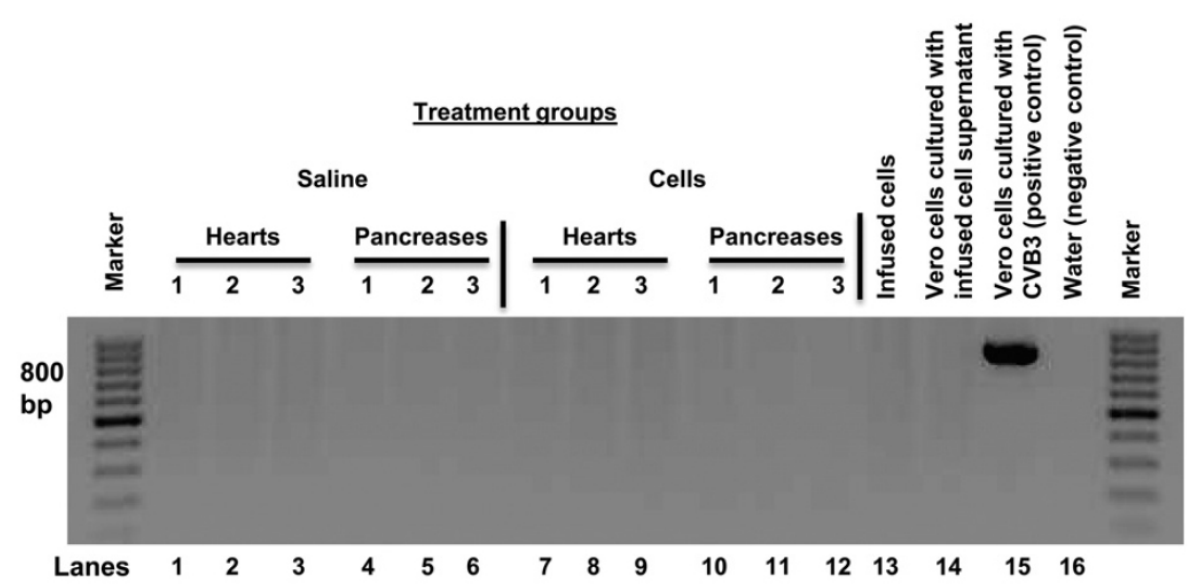

Figure 7 Detection of CVB3 RNA by RT-PCR. Total RNA was extracted from hearts and pancreases from animals that received saline or lymphocytes and other samples as indicated. After synthesizing cDNAs, PCR amplifications were performed using CVB3 VP1-specific primers. The ethidium bromide-stained PCR products were resolved in $1 \%$ agarose gel electrophoresis.

sibility, we are generating class II dextramers for other cardiac antigens such as BCKD, ANT, and TNI, which will permit us to enumerate the precursor frequencies of their $\mathrm{T}$ cell repertoires in CVB3-infected mice. These observations provide new insights into the immunopathogenesis of CVB3 infection, including therapeutic modalities.

Conflicts of interest statement

The authors declare that there are no conflicts of interest.

Acknowledgments

This work was co-funded by the American Heart Association and the Children's Cardiomyopathy Foundation (SDG2462390204001).

\section{References}

[1] P.A. Heidenreich, J.G. Trogdon, O.A. Khavjou, J. Butler, K. Dracup, M.D. Ezekowitz, E.A. Finkelstein, Y. Hong, S.C. Johnston, A. Khera, D.M. Lloyd-Jones, S.A. Nelson, G. Nichol, D. Orenstein, P.W. Wilson, Y.J. Woo, Forecasting the future of cardiovascular disease in the United States: a policy statement from the American Heart Association, Circulation 123 (2011) 933-944.

[2] R.S. Fujinami, M.G. von Herrath, U. Christen, J.L. Whitton, Molecular mimicry, bystander activation, or viral persistence: infections and autoimmune disease, Clin. Microbiol. Rev. 19 (2006) 80-94.

[3] M.B. Gravanis, N.H. Sternby, Incidence of myocarditis. A 10-year autopsy study from Malmo, Sweden, Arch. Pathol. Lab. Med. 115 (1991) 390-392.

[4] D. Cihakova, N.R. Rose, Pathogenesis of myocarditis and dilated cardiomyopathy, Adv. Immunol. 99 (2008) 95-114.

[5] D.A. Neumann, C.L. Burek, K.L. Baughman, N.R. Rose, A. Herskowitz, Circulating heart-reactive antibodies in patients with myocarditis or cardiomyopathy, J. Am. Coll. Cardiol. 16 (1990) 839-846.

[6] N. Neu, R. Klieber, M. Fruhwirth, P. Berger, Cardiac myosin-induced myocarditis as a model of postinfectious autoimmunity, Eur. Heart J. 12 (Suppl. D) (1991) 117-120.
[7] D.A. Neumann, J.R. Lane, A. LaFond-Walker, G.S. Allen, S.M. Wulff, A. Herskowitz, N.R. Rose, Heart-specific autoantibodies can be eluted from the hearts of Coxsackievirus B3-infected mice, Clin. Exp. Immunol. 86 (1991) 405412.

[8] N.R. Rose, L.J. Wolfgram, A. Herskowitz, K.W. Beisel, Postinfectious autoimmunity: two distinct phases of coxsackievirus B3-induced myocarditis, Ann. N. Y. Acad. Sci. 475 (1986) 146-156.

[9] K.S. Kim, S. Tracy, W. Tapprich, J. Bailey, C.K. Lee, K. Kim, W.H. Barry, N.M. Chapman, 5'-Terminal deletions occur in coxsackievirus B3 during replication in murine hearts and cardiac myocyte cultures and correlate with encapsidation of negative-strand viral RNA, J. Virol. 79 (2005) 7,024-7,041.

[10] M. Pauschinger, M.D. Phan, A. Doerner, U. Kuehl, P.L. Schwimmbeck, W. Poller, R. Kandolf, H.P. Schultheiss, Enteroviral RNA replication in the myocardium of patients with left ventricular dysfunction and clinically suspected myocarditis, Circulation 99 (1999) 889-895.

[11] H.S. Li, D.L. Ligons, N.R. Rose, Genetic complexity of autoimmune myocarditis, Autoimmun. Rev. 7 (2008) 168173.

[12] G. Fousteri, A. Dave, B. Morin, S. Omid, M. Croft, M.G. von Herrath, Nasal cardiac myosin peptide treatment and OX40 blockade protect mice from acute and chronic virally-induced myocarditis, J. Autoimmun. 36 (2011) 210-220.

[13] M. Estrin, S.A. Huber, Coxsackievirus B3-induced myocarditis. Autoimmunity is L3T4+ T helper cell and IL-2 independent in BALB/c mice, Am. J. Pathol. 127 (1987) 335-341.

[14] S.A. Huber, P.A. Lodge, Coxsackievirus B-3 myocarditis in Balb/c mice. Evidence for autoimmunity to myocyte antigens, Am. J. Pathol. 116 (1984) 21-29.

[15] S.A. Huber, D. Sartini, M. Exley, Vgamma4(+) T cells promote autoimmune CD8(+) cytolytic T-lymphocyte activation in coxsackievirus B3-induced myocarditis in mice: role for CD4(+) Th1 cells, J. Virol. 76 (2002) 10,78510,790 . 
[16] D.L. Donermeyer, K.W. Beisel, P.M. Allen, S.C. Smith, Myocarditis-inducing epitope of myosin binds constitutively and stably to I-Ak on antigen-presenting cells in the heart, J. Exp. Med. 182 (1995) 1,291-1,300.

[17] L.J. Reed, H. Muench, A simple method of estimating fifty percent endpoints, Am. J. Hyg. 27 (1938) 493-497.

[18] M. Afanasyeva, Y. Wang, Z. Kaya, S. Park, M.J. Zilliox, B.H. Schofield, S.L. Hill, N.R. Rose, Experimental autoimmune myocarditis in A/J mice is an interleukin-4-dependent disease with a Th2 phenotype, Am. J. Pathol. 159 (2001) 193-203.

[19] C. Massilamany, A. Gangaplara, D. Steffen, J. Reddy, Identification of novel mimicry epitopes for cardiac myosin heavy chain-alpha that induce autoimmune myocarditis in A/J mice, Cell. Immunol. 271 (2011) 438-449.

[20] C. Massilamany, S. Thulasingam, D. Steffen, J. Reddy, Gender differences in CNS autoimmunity induced by mimicry epitope for PLP 139-151 in SJL mice, J. Neuroimmunol. 230 (2011) 95-104.

[21] J. Reddy, E. Bettelli, L. Nicholson, H. Waldner, M.H. Jang, K.W. Wucherpfennig, V.K. Kuchroo, Detection of autoreactive myelin proteolipid protein 139-151-specific $\mathrm{T}$ cells by using MHC II (IAs) tetramers, J. Immunol. 170 (2003) 870-877.

[22] C. Massilamany, B. Upadhyaya, A. Gangaplara, C. Kuszynski, J. Reddy, Detection of autoreactive CD4 T cells using major histocompatibility complex class II dextramers, BMC Immunol. 12 (2011) 40.

[23] C. Massilamany, A. Gangaplara, N. Chapman, N. Rose, J. Reddy, Detection of cardiac myosin heavy chain-alphaspecific CD4 cells by using MHC class II/IA(k) tetramers in A/J mice, J. Immunol. Methods 372 (2011) 107-118.

[24] J. Wang, I.M. Okazaki, T. Yoshida, S. Chikuma, Y. Kato, F. Nakaki, H. Hiai, T. Honjo, T. Okazaki, PD-1 deficiency results in the development of fatal myocarditis in MRL mice, Int. Immunol. 22 (2010) 443-452.

[25] C. Massilamany, D. Steffen, J. Reddy, An epitope from Acanthamoeba castellanii that cross-react with proteolipid protein 139-151-reactive T cells induces autoimmune encephalomyelitis in SJL mice, J. Neuroimmunol. 219 (2010) 17-24.

[26] R.P. Loudon, A.F. Moraska, S.A. Huber, P. Schwimmbeck, P. Schultheiss, An attenuated variant of Coxsackievirus B3 preferentially induces immunoregulatory T cells in vivo, J. Virol. 65 (1991) 5,813-5,819.

[27] C. Fujimoto, C.R. Yu, G. Shi, B.P. Vistica, E.F. Wawrousek, D.M. Klinman, C.C. Chan, C.E. Egwuagu, I. Gery, Pertussis toxin is superior to TLR ligands in enhancing pathogenic autoimmunity, targeted at a neoself antigen, by triggering robust expansion of Th1 cells and their cytokine production, J. Immunol. 177 (2006) 6,896-6,903.

[28] R.K. Agarwal, S.H. Sun, S.B. Su, C.C. Chan, R.R. Caspi, Pertussis toxin alters the innate and the adaptive immune responses in a pertussis-dependent model of autoimmunity, J. Neuroimmunol. 129 (2002) 133-140.

[29] T.J. Powell, D.M. Brown, J.A. Hollenbaugh, T. Charbonneau, R.A. Kemp, S.L. Swain, R.W. Dutton, CD8+ T cells responding to influenza infection reach and persist at higher numbers than CD4+ T cells independently of precursor frequency, Clin. Immunol. 113 (2004) 89-100.
[30] K. Namba, K. Ogasawara, N. Kitaichi, N. Matsuki, A. Takahashi, Y. Sasamoto, S. Kotake, H. Matsuda, K. Iwabuchi, S. Ohno, K. Onoe, Identification of a peptide inducing experimental autoimmune uveoretinitis (EAU) in H-2Ak-carrying mice, Clin. Exp. Immunol. 111 (1998) 442-449.

[31] N. Neu, K.W. Beisel, M.D. Traystman, N.R. Rose, S.W. Craig, Autoantibodies specific for the cardiac myosin isoform are found in mice susceptible to Coxsackievirus B3induced myocarditis, J. Immunol. 138 (1987) 2,488-2,492.

[32] Z. Kaya, S. Goser, S.J. Buss, F. Leuschner, R. Ottl, J. Li, M. Volkers, S. Zittrich, G. Pfitzer, N.R. Rose, H.A. Katus, Identification of cardiac troponin I sequence motifs leading to heart failure by induction of myocardial inflammation and fibrosis, Circulation 118 (2008) 2,0632,072 .

[33] L.J. Wolfgram, K.W. Beisel, A. Herskowitz, N.R. Rose, Variations in the susceptibility to Coxsackievirus B3-induced myocarditis among different strains of mice, J. Immunol. 136 (1986) 1,846-1,852.

[34] N.R. Rose, S.L. Hill, The pathogenesis of postinfectious myocarditis, Clin. Immunol. Immunopathol. 80 (1996) S92S99.

[35] S. Huber, Tumor necrosis factor-alpha promotes myocarditis in female mice infected with coxsackievirus B3 through upregulation of CD1d on hematopoietic cells, Viral. Immunol. 23 (2010) 79-86.

[36] M. Tateyama, Mechanism of suppression of colchicine in experimental allergic encephalomyelitis, Hokkaido Igaku Zasshi 63 (1988) 72-79.

[37] M.D. Daniels, K.V. Hyland, K. Wang, D.M. Engman, Recombinant cardiac myosin fragment induces experimental autoimmune myocarditis via activation of Th1 and Th17 immunity, Autoimmunity 41 (2008) 490-499.

[38] A. Jager, V. Dardalhon, R.A. Sobel, E. Bettelli, V.K. Kuchroo, Th1, Th17, and Th9 effector cells induce experimental autoimmune encephalomyelitis with different pathological phenotypes, J. Immunol. 183 (2009) 7,1697,177 .

[39] Y. Fan, W. Weifeng, Y. Yuluan, K. Qing, P. Yu, H. Yanlan, Treatment with a neutralizing anti-murine interleukin-17 antibody after the onset of coxsackievirus b3-induced viral myocarditis reduces myocardium inflammation, Virol. J. 8 (2011) 17.

[40] M.A. Opavsky, J. Penninger, K. Aitken, W.H. Wen, F. Dawood, T. Mak, P. Liu, Susceptibility to myocarditis is dependent on the response of alphabeta $\mathrm{T}$ lymphocytes to coxsackieviral infection, Circ. Res. 85 (1999) 551-558.

[41] A. Henke, S. Huber, A. Stelzner, J.L. Whitton, The role of CD8+ T lymphocytes in coxsackievirus B3-induced myocarditis, J. Virol. 69 (1995) 6,720-6,728.

[42] H.S. Domingues, M. Mues, H. Lassmann, H. Wekerle, G. Krishnamoorthy, Functional and pathogenic differences of Th1 and Th17 cells in experimental autoimmune encephalomyelitis, PLoS One 5 (2010) e15531.

[43] D. Fairweather, S. Frisancho-Kiss, S.A. Yusung, M.A. Barrett, S.E. Davis, R.A. Steele, S.J. Gatewood, N.R. Rose, IL-12 protects against coxsackievirus B3-induced myocarditis by increasing IFN-gamma and macrophage and neutrophil populations in the heart, J. Immunol. 174 (2005) 261,269. 
[44] M.S. Horwitz, A. La Cava, C. Fine, E. Rodriguez, A. Ilic, N. Sarvetnick, Pancreatic expression of interferon-gamma protects mice from lethal coxsackievirus B3 infection and subsequent myocarditis, Nat. Med. 6 (2000) 693-697.

[45] J. Li, S. Leschka, S. Rutschow, P.L. Schwimmbeck, L. Husmann, M. Noutsias, D. Westermann, W. Poller, H. Zeichhardt, K. Klingel, C. Tschope, H.P. Schultheiss, M. Pauschinger, Immunomodulation by interleukin-4 suppresses matrix metalloproteinases and improves cardiac function in murine myocarditis, Eur. J. Pharmacol. 554 (2007) 60-68.

[46] P. Hofmann, M. Schmidtke, A. Stelzner, D. Gemsa, Suppression of proinflammatory cytokines and induction of IL-10 in human monocytes after coxsackievirus B3 infection, J. Med. Virol. 64 (2001) 487-498.

[47] A. Henke, C. Mohr, H. Sprenger, C. Graebner, A. Stelzner, M. Nain, D. Gemsa, Coxsackievirus B3-induced production of tumor necrosis factor-alpha, IL-1 beta, and IL-6 in human monocytes, J. Immunol. 148 (1992) 2,270-2,277.

[48] Y. Seko, N. Takahashi, H. Yagita, K. Okumura, Y. Yaza$\mathrm{ki}$, Expression of cytokine mRNAs in murine hearts with acute myocarditis caused by coxsackievirus b3, J. Pathol. 183 (1997) 105-108.
[49] G.C. Baldeviano, J.G. Barin, M.V. Talor, S. Srinivasan, D. Bedja, D. Zheng, K. Gabrielson, Y. Iwakura, N.R. Rose, D. Cihakova, Interleukin-17A is dispensable for myocarditis but essential for the progression to dilated cardiomyopathy, Circ. Res. 106 (2010) 1,646-1,655.

[50] D. Fairweather, N.R. Rose, Coxsackievirus-induced myocarditis in mice: a model of autoimmune disease for studying immunotoxicity, Methods 41 (2007) 118-122.

[51] S. Tracy, K. Hofling, S. Pirruccello, P.H. Lane, S.M. Reyna, C.J. Gauntt, Group B coxsackievirus myocarditis and pancreatitis: connection between viral virulence phenotypes in mice, J. Med. Virol. 62 (2000) 70-81.

[52] H. Nakamura, T. Yamamoto, T. Yamamura, F. Nakao, S. Umemoto, T. Shintaku, K. Yamaguchi, P. Liu, M. Matsuzaki, Repetitive coxsackievirus infection induces cardiac dilatation in post-myocarditic mice, Jpn. Circ. J. 63 (1999) 794-802.

[53] M.W. Cunningham, T cell mimicry in inflammatory heart disease, Mol. Immunol. 40 (2004) 1,121-1,127.

[54] E.J. McMahon, S.L. Bailey, C.V. Castenada, H. Waldner, S.D. Miller, Epitope spreading initiates in the CNS in two mouse models of multiple sclerosis, Nat. Med. 11 (2005) 335-339. 